\title{
Cultural and learning differences in the Judd illusion
}

\author{
John van der Kamp • Rob Withagen • \\ Matthieu M. de Wit
}

Published online: 11 April 2013

(C) Psychonomic Society, Inc. 2013

\begin{abstract}
In the present study, we examined whether individual differences in the perception of illusory Judd drawings point to variability in the pickup of informational variables. Two sources for these individual differences were addressed: culture and learning. East Asian $(n=24)$ and Western $(n=24)$ participants made perceptual judgments of the midpoint of the shaft of various Judd figures in a pretestpractice-posttest design. During practice, half of the participants received feedback about the actual midpoint after each trial, while the other half did not receive feedback. The results showed differences among perceivers of different cultures in judging the midpoints of the shafts of Judd figures, particularly with respect to their propensity to improve perceptual accuracy after repeated practice and feedback. For most participants, changes in illusory bias as a consequence of learning were shown to reflect either a change in what informational variable they exploited or a rescaling or calibration of the perception to the informational variable. However, the individual differences in illusory bias related to culture could not be unequivocally attributed to either of these perceptual-learning processes.
\end{abstract}

Keywords Perception - Individual differences · Culture . Ecological psychology $\cdot$ Attunement $\cdot$ Calibration $\cdot$ Attention in learning $\cdot$ Perceptual learning

\footnotetext{
J. van der Kamp $(\square)$

Research Institute MOVE Amsterdam, Faculty of Human

Movement Sciences, VU University Amsterdam,

Van der Boechorststraat 9,

1081 BT Amsterdam, The Netherlands

e-mail: j_van_der_kamp@fbw.vu.nl

J. van der Kamp • M. M. de Wit

Institute of Human Performance, University of Hong Kong,

Hong Kong, SAR, China

R. Withagen

Center for Human Movement Sciences, University Medical Center

Groningen, Groningen, The Netherlands
}

Individual differences abound in human perception. On occasion, for example, perception of the environment is not alike between individuals of different cultures (e.g., Nisbett, 2003; Segall, Campbell, \& Herskovits, 1966). It astonishes us, therefore, that mainstream theories of human perception presume that perception is more or less universal, and are often directed toward an understanding of how, in general, human beings perceive the world (for an extensive critique, see Henrich, Heine, \& Norenzayan, 2010). We believe that any theoretical approach to human perception should also acknowledge individual differences, because perceptual systems have evolved, and they develop significantly during a life span. Ecological psychology offers an approach that can take into account that perceptual systems have different histories adapting to their environments (e.g., Chemero, 2009; Gibson, 1966, 1979; Ingold, 2000; Reed, 1996; Rosengren, Savelsbergh, \& van der Kamp, 2003; Warren, 2005; Withagen \& Chemero, 2009).

In the present study, we assessed individual differences in the perception of geometrical illusions among individuals of different cultures from an ecological perspective. There is a long, but controversial, tradition of research that has (purportedly) demonstrated that individuals from different cultures vary in their susceptibility to illusory drawings (e.g., Rivers, 1905; Segall et al., 1966; see also de Fockert, Davidoff, Fagot, Parron, \& Goldstein, 2007; Ji, Peng, \& Nisbett, 2000). Most well-known is the work by Segall et al., which showed that perceivers from small-scale, mostly African, cultures with few "carpentered," rectangular structures in their environments are less susceptible to the Müller-Lyer illusion and more susceptible to the horizontalvertical illusion than are Westerners. In fact, in an early article that compared the biases for these illusory drawings between Papuan people from New Guinea and English persons, Rivers had already anticipated these differences. Intriguingly, Rivers wrote, with respect to the Müller-Lyer illusion, that the variability in perception occurs because of "a difference in the direction of attention, the savage 
attending more strictly to the two lines which he is desired to make equal, while the civilised man allows the figure as a whole to exert its full influence on his mind" (p. 363). A similar view reverberates in Gibson's (1966) suggestion that "only if we can isolate the two line segments from the wings and arrowheads in the Müller-Lyer illusion should they appear equal, and this would require a very special kind of selective attention" (p. 313).

In the present context, such differences in attention would point to differences in the pickup of informational variables. That is, ecological psychologists have explained differences in perceptual accuracy in terms of the detected informational variables (e.g., Jacobs, Runeson, \& Michaels, 2001; Michaels \& de Vries, 1998; Withagen \& van Wermeskerken, 2009). The idea is that informational variables differ in their degrees of usefulness (e.g., Jacobs \& Michaels, 2007; Withagen, 2004; Withagen \& van der Kamp, 2010). Specifying variables, on the one hand, relate one-to-one to the to-be-perceived property; nonspecifying variables, on the other hand, relate ambiguously to that property. This implies that biases in perceptual judgments can be accounted for in terms of the pickup of nonspecifying informational variables. ${ }^{1}$ Hence, in the case of the Müller-Lyer figure, for instance, the use of optical variables that comprise contextual features, such as the fins (i.e., nonspecifying variables), will result in the illusory bias, while directing attention to an informational variable that specifies the length of the line itself should allow for accurate perception. Until now, however, cross-cultural research (or any other domain of research in which differences in the perception of illusions have been investigated) has not focused on individual differences in the pickup of optic variables. However, observers who use nonspecifying variables may show different degrees of illusory bias (dependent on the particular variable that they exploit), while observers who rely on specifying variables would not show a bias at all. Hence, because previous work has only reported group averages, we cannot distinguish from this work whether the cross-cultural differences reflect differences in the degrees to which the perception of members of different cultures are biased or differences in the proportions of individuals of a culture who are susceptible to the illusion.

The purpose of the present study was therefore to assess whether cross-cultural differences in the perception of illusions reflect differences in the pickup of informational variables. To this end, we investigated the perception of the midpoint of the line in Judd drawings (Fig. 1) among East

\footnotetext{
${ }^{1}$ To be clear, we mean nonspecifying to the property of interest. The same variable can be, and most likely is, specific to some other property of the environment, or even to the property of interest under different environmental constraints (see Runeson, 1988).
}
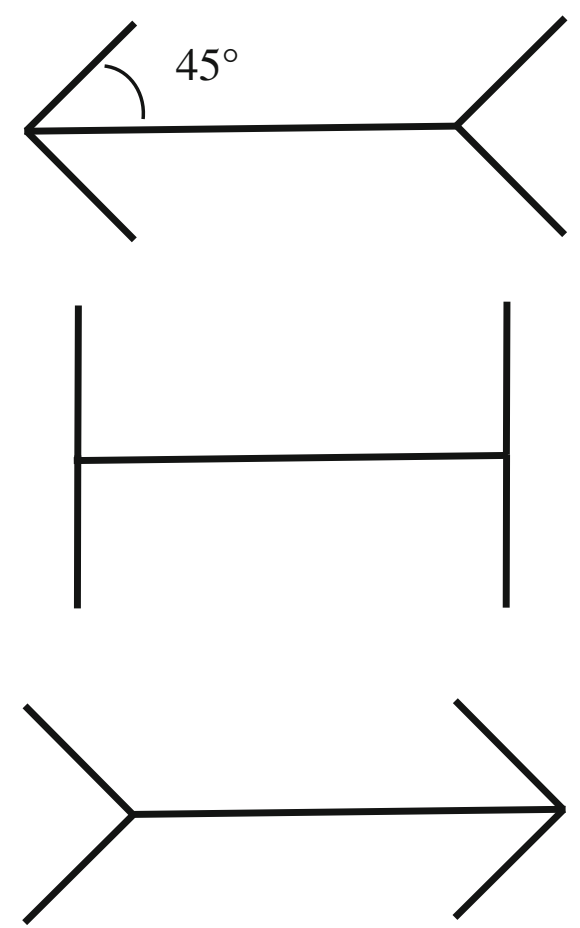

Fig. 1 The left-fin, neutral, and right-fin Judd figures (top to bottom) used in the pretest and posttest (to scale)

Asians and Westerners. The Judd drawing is a variant of the much-used Müller-Lyer illusion. Typically, the perceived midpoint of the line in a Judd drawing is biased in the direction opposite to the side to which the arrow-like fins point. Nisbett (2003, 2007; Nisbett \& Miyamoto, 2005) has argued that, in reasoning about and perceiving objects and events, East Asians attend more toward contextual aspects of objects in drawings and pictures. By contrast, Western people focus more on the objects, and relative to East Asian people, tend to ignore the contextual aspects (see also Ames \& Fiske, 2010). Ji et al. (2000), for example, showed that East Asians (mostly Chinese) were more prone to perceptual inaccuracies in the rod-and-frame illusion than were Americans. Following this work, we hypothesized that the illusory bias for Judd drawings would be larger among East Asian (Hong Kong Chinese) perceivers than among Western (Dutch) perceivers. ${ }^{2}$ Alongside this, we expected that the use of nonspecifying informational variables, which comprise the contextual illusion-inducing aspects of the Judd drawing (i.e., the angle and size of the fins; see, e.g., Jacobs, Ibáñez-Gijón, Díaz, \& Travieso, 2011; Massaro \& Anderson, 1970; Pressey, 1974), would be more prevalent among the East Asian perceivers.

\footnotetext{
${ }^{2}$ A developmental study by Dawson, Young, and Choi (1973), in fact, reported a smaller(!) illusory bias for the Müller-Lyer illusion among East Asian adults than among Western adults. However, the authors did not collect the Western data themselves, but used the samples of Segall et al. (1966) for the purpose of a cross-cultural comparison.
} 
Recently, Danks and Rose (2010) argued that while perceptions may indeed differ across individuals of different cultures, the underlying perceptual learning processes are likely to be the same. This raises the issue of interindividual differences in the capacity for perceptual learning. Over the last decade, several ecologically motivated studies have reported considerable individual differences in perceptual learning in Western participants (e.g., Jacobs, Michaels, \& Runeson, 2000; Jacobs et al., 2001; Menger \& Withagen, 2009; Michaels \& de Vries, 1998; Runeson \& Andersson, 2007; Withagen \& van Wermeskerken, 2009). Participants have been found to vary in the degrees to which they were able to take advantage of feedback in order to attune to more useful informational variables (Withagen \& van Wermeskerken, 2009). It was also found that this capacity to attune declines in the elderly (Withagen \& Caljouw, 2011). Accordingly, variability in illusory bias among groups of individuals may point to different aptitudes to attune - attunement being the process of convergence from less to more useful informational variables.

In order to explore cross-cultural differences in perceptual learning, we employed a pretest-practice-posttest design. Half of the participants received feedback during practice, and the other half did not. Although ample evidence has shown that practice-even without feedback - may reduce illusory bias (e.g., for the Müller-Lyer illusion; see Eysenck \& Slater, 1958; Judd, 1902; Schiano \& Jordan, 1990), it has not been directly tested whether this is due to a convergence to specifying informational variables. A recent article by Jacobs et al. (2011), however, has provided the proverbial exception: The authors found a marginal reduction in the illusory bias in the perception of the length of the shaft in the Müller-Lyer illusion after a short period of practice with veridical feedback, and suggested that this was likely due to a stronger reliance on length-specifying information ${ }^{3}$; yet, differences in perceptual accuracy or learning between the participants were not considered. Hence, we hypothesized that a reduction or disappearance of the illusory bias with practice would most likely result from a shift in picking up nonspecifying variables toward a reliance on more useful variables. Given their propensity for ignoring contextual aspects, we also explored whether Westerners would be more prone to show attunement than would East Asians.

\footnotetext{
${ }^{3}$ Jacobs et al. (2011) portrayed perceptual learning in dynamical terms as a travelling on a path in an information space, with each point in space representing an informational variable. Roughly, the minimum value in this space represents the variable that is specific to the shaft length of the Müller-Lyer illusion (e.g., optical angle), while higher values represent nonspecifying variables related to combinations of the fins' lengths and angles. Jacobs et al. (2011) showed that the value in informational space decreases as a consequence of practice, suggesting that participants came to rely on more useful informational variables.
}

\section{Method}

\section{Participants}

Groups of 24 Western Caucasian students (13 female, 11 male), from the University of Groningen, the Netherlands, and 24 East Asian Hong Kong Chinese students (9 female, 15 male), from the University of Hong Kong, Hong Kong SAR, volunteered to participate in the experiment. The participants ranged from 20 to 40 years of age, were all righthanders (i.e., the illusory bias in perception tasks may differ between right- and left-handers; see, e.g., van der Kamp, de Wit, \& Masters, 2012), and had normal or corrected-to-normal vision. The participants received a small monetary fee for their cooperation and were naive as to the purpose of the experiment. The study was conducted in accordance with the ethical guidelines of both institutions.

\section{Apparatus}

The stimuli were presented on a 15 -in. computer screen. Two sets of blue stimuli on a white background were created in and presented with MATLAB (MathWorks, Natick, MA). The stimulus sets for the pre- and posttests consisted of three types: a left-fin Judd figure, a right-fin Judd figure, and a neutral Judd figure. Each of these figures had a shaft length of $4.0 \mathrm{~cm}$ and fin lengths of $1.4 \mathrm{~cm}$, while the angles between the fin and the shaft were $45^{\circ}, 135^{\circ}$, and $90^{\circ}$ for the left, right, and neutral Judd figures, respectively. The stimulus set for the practice phase consisted of six types - that is, three pairs of left-fin and right-fin Judd figures with shaft lengths of $8.0 \mathrm{~cm}$ and different fin measures. One pair had fins $4.2 \mathrm{~cm}$ in length, angled $45^{\circ}$ and $135^{\circ}$ to the shaft for the left and right Judd figures, respectively; the second pair had same-length fins of $4.2 \mathrm{~cm}$ and more acute angles of $32^{\circ}$ and $148^{\circ}$; while the third pair had both shorter fins of $2.8 \mathrm{~cm}$ and more acute angles of $32^{\circ}$ and $148^{\circ}$. The larger stimuli for the practice phase were chosen because they were likely to induce a larger absolute illusory bias (Greene \& Nelson, 1997), and therefore were thought to enhance impetus for learning. Furthermore, using different stimuli sets in practice blocks and tests blocks may provide additional hints as to the nature of the learning process (see the Discussion section for a more elaborate argument). At the start of each trial, a small red vertical marker of $0.3 \mathrm{~cm}$ was positioned within $1.0 \mathrm{~cm}$ of one of the endpoints on the line. By pressing the left and right arrow keys on the keyboard, the participants could move the marker along the Judd figures' line toward the midpoint in steps of $0.25 \mathrm{~mm}$. During the practice phase, knowledge of results (KR) was provided at the end of each trial via a green vertical marker of $1.0 \mathrm{~cm}$ at the exact midpoint of the line. In other words, at the end of each trial, both the perceived midpoint (i.e., red marker) and the actual midpoint (i.e., green marker) 
were visible to the participant (only for the feedback groups; see below).

\section{Procedure and design}

Participants were seated behind the computer at a comfortable distance. The Hong Kong Chinese participants made up the East Asian group, whereas the Dutch participants formed the Western group. Half of the participants in each group were randomly assigned to the feedback condition, in which KR was provided during practice, while the other half were allocated to the no-feedback condition. A pretestpractice-posttest design was employed. During the preand posttests, participants were presented with each of three Judd figures (i.e., left-fin, right-fin, and neutral) six times in a randomized order, resulting in a total of 18 trials. The figures were displayed with their midpoints at one of three locations relative to the center of the screen (i.e., with the figure midpoint $1.0 \mathrm{~cm}$ to the right or left of the screen's center, or exactly at the center). The order of presentation was the same in the pre- and posttests. A trial started with the presentation of the figure with the small red marker. The participant was instructed to move the red marker to the exact midpoint of the line by pressing the right and left arrow keys on the keyboard. Participants were allowed to correct their initial estimates, and no time requirements were instigated. Yet, they were told not to use their hands or fingers or any tools to "measure" the midpoint. ${ }^{4}$ Participants pressed the Enter key once they were satisfied with the position of the red marker. This stored the position of that marker and triggered the subsequent trial.

The practice phase consisted of five blocks of 36 trials, during which the six Judd figures were presented six times in a randomized order, which differed for each block. The figures were displayed at the same three locations relative to the center of the screen as in the pre- and posttests. For the no-feedback groups, the procedure was similar to that of the pre- and posttests. The feedback groups, however, received $\mathrm{KR}$ at the end of each trial. That is, at the end of the trial, besides the red marker (i.e., indicating the participants' judgments of the line midpoints), the green marker (i.e., indicating the actual midpoint of the line) was shown. Participants were told that the difference between the two markers represented the magnitude and direction of the error in perceived midpoint. They were told to try to nullify the error. Hence, participants received KR, but they were not instructed as to how they should use the feedback to reduce their error. KR ended after the participants pressed the Enter key again, to trigger the next trial. Participants in both the feedback and no-feedback groups were told that the most

\footnotetext{
${ }^{4}$ One East Asian participant in the no-feedback group did not comply with these instructions and was excluded from the analysis.
}

accurate participant would gain an additional prize of approximately $€ 25$. The prize was awarded in each of the four groups. A short, 1-min break followed every practice block, and a 5-min break followed the pretest and the fifth and final practice block.

\section{Data analysis}

For each trial, we calculated the signed-error score (in millimeters), defined as the distance between the perceived and actual midpoints of the shaft. Participants were very accurate for the neutral, nonillusory Judd figure; for all four groups, $t$ tests showed that neither the error score in the pretest nor the error in the posttest exceeded zero (all $p \mathrm{~s}>$ .15) with these figures. Hence, we simplified the analyses by using the illusory bias as the main dependent variable - that is, the difference between the signed-error scores for the leftand right-fin Judd figures. We then compared the magnitudes of the illusory bias for the pre- and posttests and for the practice phase as a function of culture and feedback. To this end, the corresponding illusory bias scores were submitted to two separate analyses of variance, with Culture (East Asian, Western) and Feedback (feedback, no feedback) as between-subjects factors, and either Test (pretest, posttest) or Block (Practice Block 1-5) as a within-subjects factor. In the case of a violation of the sphericity assumption, Huynh-Feldt corrections of the $p$ values are reported. Tukey HSD tests were used to compare differences between the means, and $\eta^{2}$ was used as the measure of effect size.

In addition, to address whether changes in the illusory bias during the practice phase and from the pretest to the posttest reflected changes in the informational variable that was used, we determined the degree to which illusioninducing aspects of the Judd figures were associated with the signed-error scores. A strong correlation would point to the use of nonspecifying informational variables in the perception of the midpoint of the line. (A strong correlation between the actual midpoint and the signed-error scores would have been consistent with the use of a specifying informational variable. However, we were unable to assess this correlation, because we presented only one shaft length, and hence one midpoint position, within tests and blocks.) In particular, for each individual participant for the pretest, posttest, and each of the five practice blocks, we calculated Pearson product-moment correlations between the signederror score and the size of the angle between the fins and the shaft of the Judd figures. ${ }^{5}$ In order to compare differences in information pickup, the $r$ values were arcsine-transformed

\footnotetext{
5 The same correlation analysis was performed for the length of the fins. However, almost no significant correlations were revealed, suggesting that in the present study, its explanatory power was negligible. Therefore, we do not present the details of this analysis.
} 
and submitted to separate analyses of variance with Culture (East Asian, Western) and Feedback (feedback, no feedback) as between-subjects factors and either Test (pretest, posttest) or Block (Practice Block 1-5) as the withinsubjects factor. Test and practice blocks were not directly compared, because $r$ values were calculated from different numbers of observations.

\section{Results}

Pretest and posttest comparisons

Figure 2 displays the magnitudes of illusory bias in the pretest and posttest (i.e., before and after practice). As anticipated, the illusory bias only reduced for the groups that received feedback during practice. Accordingly, the analysis of variance revealed significant effects of test, $F(1,43)=75.3, p<.001, \eta^{2}=.64$, and feedback, $F(1,43)$ $=16.7, p<.001, \eta^{2}=.28$, and a Test $\times$ Feedback interaction, $F(1,43)=39.4, p<.001, \eta^{2}=.48$. Post hoc tests indicated that the feedback groups significantly reduced the illusory bias from the pre- to the posttest, while the no-feedback groups did not. In fact, one-sample $t$ tests showed that after practice with feedback, the bias did not significantly differ from zero, $p \mathrm{~s}>.6$. The analysis of variance also revealed a marginally significant effect of culture, $F(1,43)=3.70, p=.06, \eta^{2}=.08$, and a significant Culture $\times$ Feedback interaction, $F(1,43)=5.05$, $p<.05, \eta^{2}=.11$. Post hoc tests indicated that for the no-feedback groups, the illusory bias was larger among the East Asians $(2.8 \mathrm{~mm})$ than among the Westerners $(1.8 \mathrm{~mm})$, while for the feedback group, the difference between East Asians $(1.3 \mathrm{~mm})$ and Westerners $(1.4 \mathrm{~mm})$ did not reach significance. This interaction effect was not mediated by test.

\section{Practice block comparisons}

Figure 3 shows the magnitudes of the illusory bias during the five practice blocks. Notice that the bias during practice was larger than during the pretests, particularly for the no-feedback groups (cf. Fig. 2). This can easily be attributed to the use of larger Judd figures in the practice blocks. Figure 3 demonstrates clear differences in illusory bias between practice blocks, feedback groups, and East Asians and Westerners. This was confirmed by significant main effects of block, $F(4,172)=13.7, p<.001, \eta^{2}=.24$, feedback, $F(1,43)=$ $44.8, p<.001, \eta^{2}=.51$, and culture, $F(1,43)=6.87, p<.05$, $\eta^{2}=.14$. Yet, these effects were qualified by a significant Block $\times$ Feedback $\times$ Culture interaction, $F(4,172)=3.26, p<$ $.05, \eta^{2}=.07$. Post hoc tests indicated that both the East Asian and Western feedback groups significantly
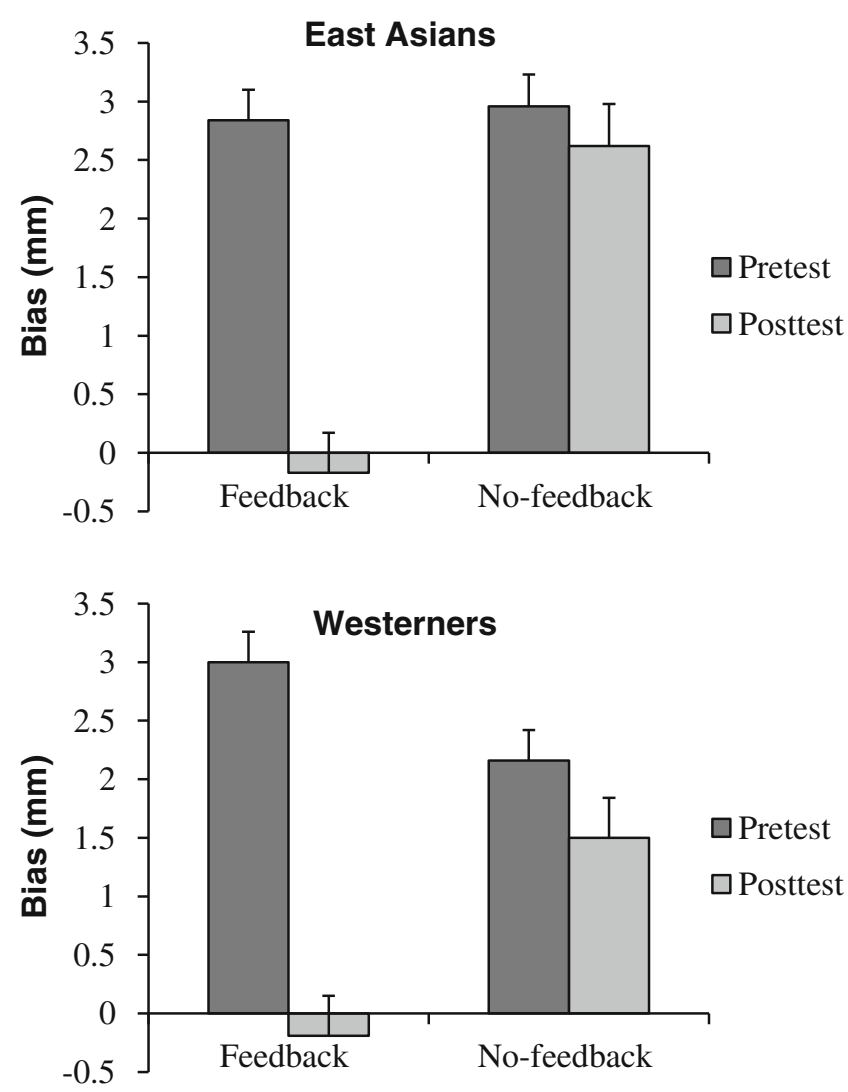

Fig. 2 Illusory bias in millimeters (with bars representing $S E$ s), as a function of test and feedback for East Asians (top panel) and Westerners (bottom panel)

reduced their illusory bias during practice. However, the East Asians did so less rapidly. This was indicated by a significantly larger bias in the first two practice blocks for the East Asians than for the Westerners. Yet, one-sample $t$ tests showed that even in the final practice block, the illusory bias remained significant for both groups $(p s<.05)$. In addition, post hoc test indicated that throughout each of the five practice blocks, both feedback groups showed significantly smaller illusory biases than did the two no-feedback groups. The observation that this included the first block indicates that a significant reduction had already occurred early in practice. Finally, whereas the East Asian no-feedback group did not show a significant drop in the magnitude of the illusory bias, a significant reduction of the bias did occur among the Western no-feedback group. Hence, without feedback, the illusory bias was consistently larger for East Asians than for Westerners.

Information pickup during practice and from pretest to posttest

In order to build the argument concisely, we present the results with respect to informational variable use during 


\section{Feedback}
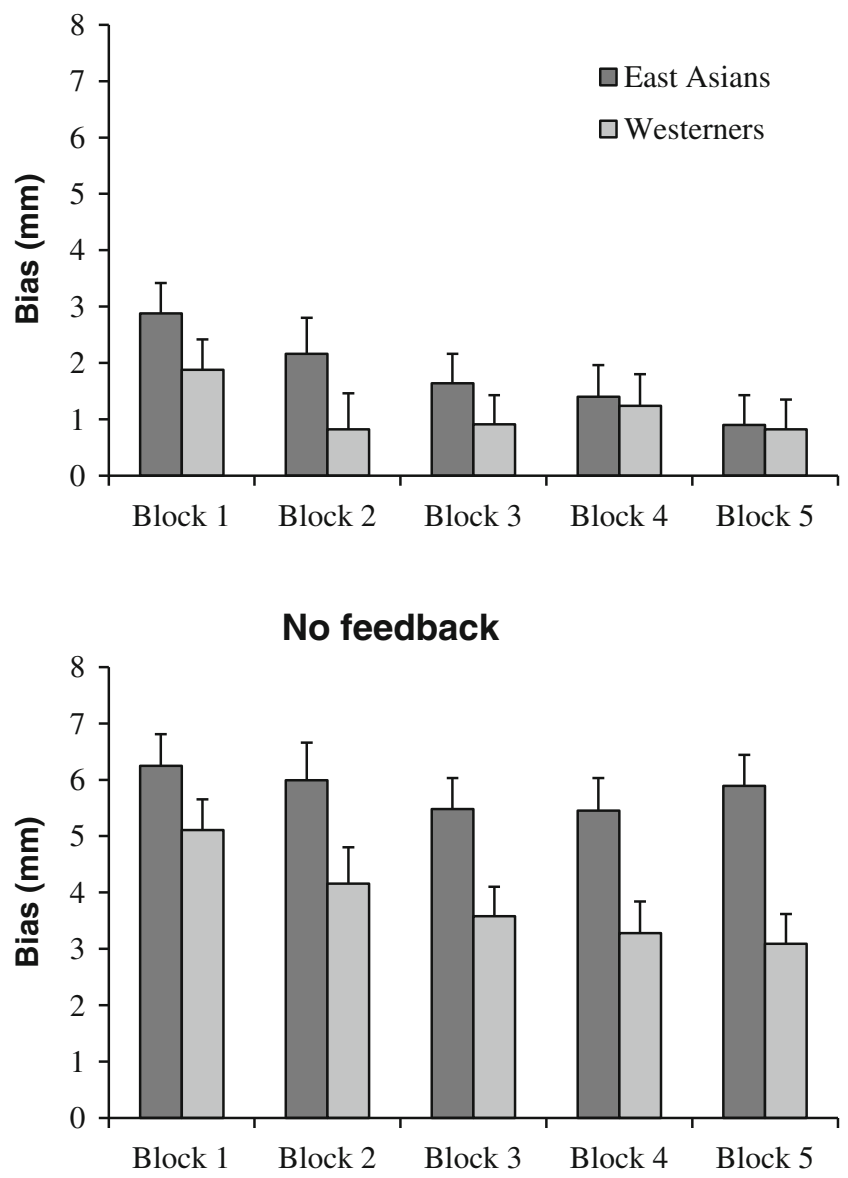

Fig. 3 Illusory bias in millimeters (with bars representing $S E$ s), as a function of culture and practice block for the feedback group (top panel) and the no-feedback group (bottom panel)

practice first, and then discuss the pre- and posttests. Figure 4 charts the use of nonspecifying illusion-inducing information during the five practice blocks for a selection of individual participants. It does so by displaying the Pearson correlations between the signed-error score (i.e., the difference between perceived and actual midpoints) and the illusion-inducing aspects of the Judd figures (i.e., the sizes of the angle between the fins and the vertical line). Significant correlations (i.e., correlations that do not fall between the two dashed horizontal lines in the figures), together with a considerable illusion bias, suggest that a nonspecifying variable was exploited that related to the illusion-inducing aspects of the Judd figure. ${ }^{6}$ For many of the participants in the feedback groups, the $r$ values decreased during practice (see, e.g., participants EA2, EA4, EA7, W1, W3, W6, and W7 in Fig. 4). In the fifth and final practice block, a total of

\footnotetext{
$\overline{{ }^{6}}$ The assumption that high significant correlations point to the use of a nonspecifying illusion-inducing variable is supported by highly significant correlations between the $r$ values (i.e., after arcsine transformation) and the magnitudes of the illusory bias for Practice Blocks 1-5 separately, $r \mathrm{~s}(47)>.83, p \mathrm{~s}<.001$.
}

six out of 12 East Asians (e.g., EA7) and seven out of 12 Westerners (e.g., W1, W3, W6, and W7) showed nonsignificant correlations between the signed-error score and the illusion-inducing aspects, indicating that they abandoned using the nonspecifying informational variable, possibly attuning to specifying or to other nonspecifying, but more useful, informational variables. ${ }^{7}$ These participants all had a negligible illusory bias of less than $1.0 \mathrm{~mm}$ by the end of practice. (Note the contrast with the analysis for the group as whole, which had suggested a significant bias; see the Practice block comparisons section.) Notice that EA4 showed a significant negative correlation in the fifth practice block. This participant appears to have still used the nonspecifying informational variable, but scaled it differently to perception (i.e., rather than the midpoint of the line being perceived in the direction opposite to the side to which the arrow-like fins pointed, the midpoint was now perceived in the direction on the same side to which the arrow-like fins pointed). Indeed, he showed a negative illusory bias in this final practice block.

By contrast, the vast majority of the participants in the no-feedback groups showed high $r$ values throughout the practice blocks (e.g., EA15, EA17, EA22, EA24, W14, W20, and W24), together with a considerable illusory bias. This strongly indicates that they stuck to the nonspecifying informational variable that they used in the first block and did not converge to other variables during practice. There were, however, two exceptions among the Westerners (e.g., W19). They both showed dramatic decrements in $r$ values in the final practice block. This coincided with the disappearance of the illusory bias, suggesting that they might have attuned to a specifying informational variable, even though they did not receive feedback.

Figure 5 presents the average Pearson correlations for each group as a function of practice block. An analysis of variance on the arcsine-transformed correlations substantiated significant main effects of block, $F(4,172)=$ 8.03, $p<.001, \eta^{2}=.16$, and feedback, $F(1,43)=85.3$, $p<.001, \eta^{2}=.68$. Post hoc tests indicated significant decreases in $r$ values from the first to the second and from the fourth to the final practice block, with the $r$ values for the feedback groups being significantly lower than those for the no-feedback groups. However, we observed no significant effects of culture. [A one-way ANOVA comparing the correlations for the final practice blocks of the East Asian and Western no-feedback groups failed to reach significance, $F(1,22)=3.48$, $p=.07$.

${ }^{7}$ In fact, five of these participants already showed nonsignificant $r \mathrm{~s}$ in the first practice block. Because they all showed highly significant $r \mathrm{~s}$ in the pretest (see below), it is likely that these participants attuned to a more useful variable after only a few trials with feedback. 
Fig. 4 Pearson correlations between signed-error scores and the size of the angle between the fins and the shaft of the Judd figures, as a function of practice block for a subset of the individual participants in the East Asian feedback group (top left panel), the East Asian nofeedback group (top right panel), the Western feedback group (bottom left panel), and the Western no-feedback group (bottom right panel)
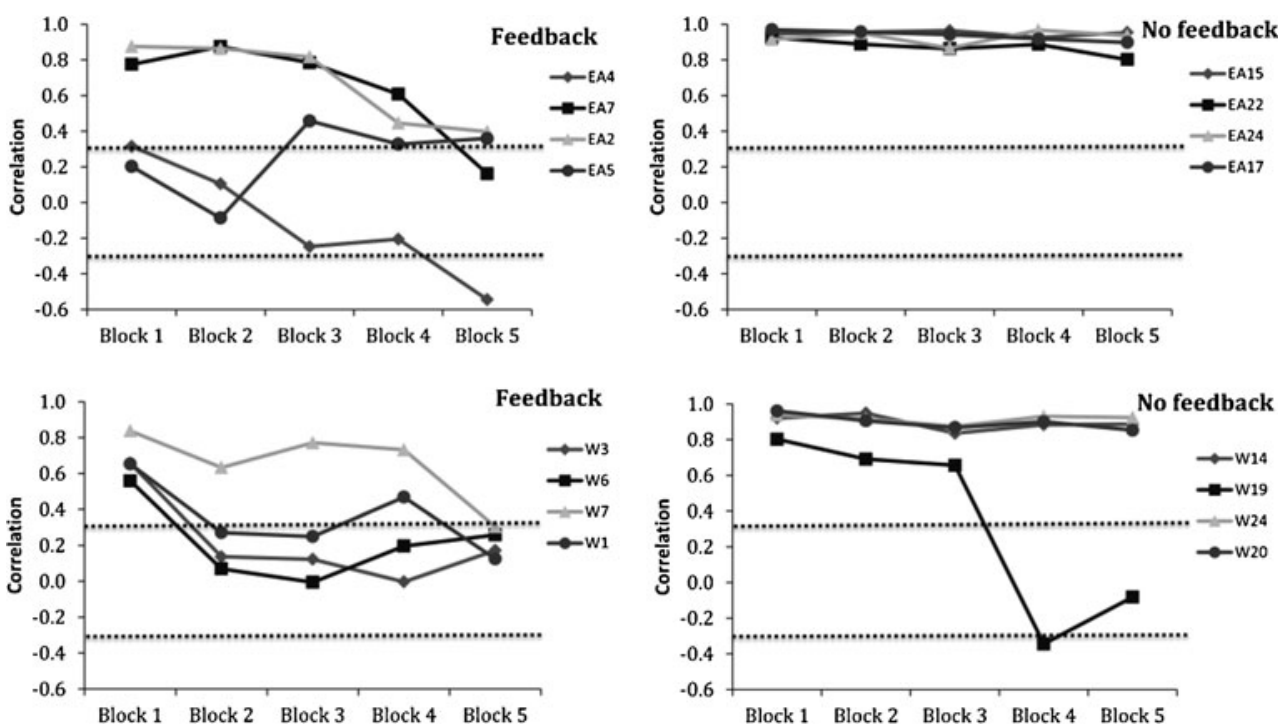

The average Pearson correlations for the pre- and posttests are depicted in Fig. 6. The $r$ values decrease from the pretest to the posttest, but only for the two groups that received feedback. Accordingly, significant effects of test, $F(1,43)=61.2$, $p<.001, \eta^{2}=.59$, and feedback, $F(1,43)=22.4, p<.001$, $\eta^{2}=.34$, as well as a Test $\times$ Feedback interaction, $F(1,43)=$ $29.0, p<.001, \eta^{2}=.40$, were found. No effects of culture were revealed. Yet, Fig. 7, which presents the correlations for a selection of individual participants (cf. Fig. 4), shows that the differences in variable use may be much larger than are suggested by the group averages, particularly for the posttest. For the pretest, the high $r$ values seem to mirror the group averages; participants' perceptions of the midpoint were clearly consistent with the use of the nonspecifying informational variable related to the illusion-inducing aspect of the Judd figure. The posttest also showed high $r$ values among the participants in the no-feedback groups (and considerable illusory biases): These participants kept using the same nonspecifying informational variable. One exception was apparent among the Western participants (W19). Her low correlation in the posttest might point to a spontaneous convergence to a specifying variable. Accordingly, her illusory bias had become negligible $(0.3 \mathrm{~mm})$.
The differences in variable use in the posttest among participants of the feedback groups are more intricate. In total, two out of 12 East Asian (e.g., EA5) and four out of 12 Western (e.g., W6 and W1) participants showed low $r$ values in the posttest. This indicates that feedback led these participants to attune to a different informational variable, even after the feedback had been withdrawn. A minor illusory bias (i.e., less than $1.0 \mathrm{~mm}$ ) suggests that they might have homed in on using a specifying variable. For a second group of participants in the feedback groups, among whom were five East Asians (e.g., EA2) and four Westerners (e.g., W7), feedback did not seem to have resulted in a permanent shift away from the use of the nonspecifying informational variables. These participants had significant positive correlations in the posttest, which occurred even though some of the participants (e.g., W7; cf. Fig. 5) had apparently converged to a variable other than the nonspecifying variable related to the illusioninducing aspects of the Judd figure in the final practice block; it seems that this attunement was not maintained in the posttest. Finally, the remaining five East Asian (e.g., EA4 and EA7) and four Western (e.g., W3) participants showed a significant, but negative, correlation in the posttest. Interestingly, each of these participants showed a negative and mostly
Fig. 5 Average Pearson correlations (with bars representing $S E$ s) between signed-error scores and the size of the angle between the fins and the shaft of the Judd figures, as a function of culture and feedback for each practice block

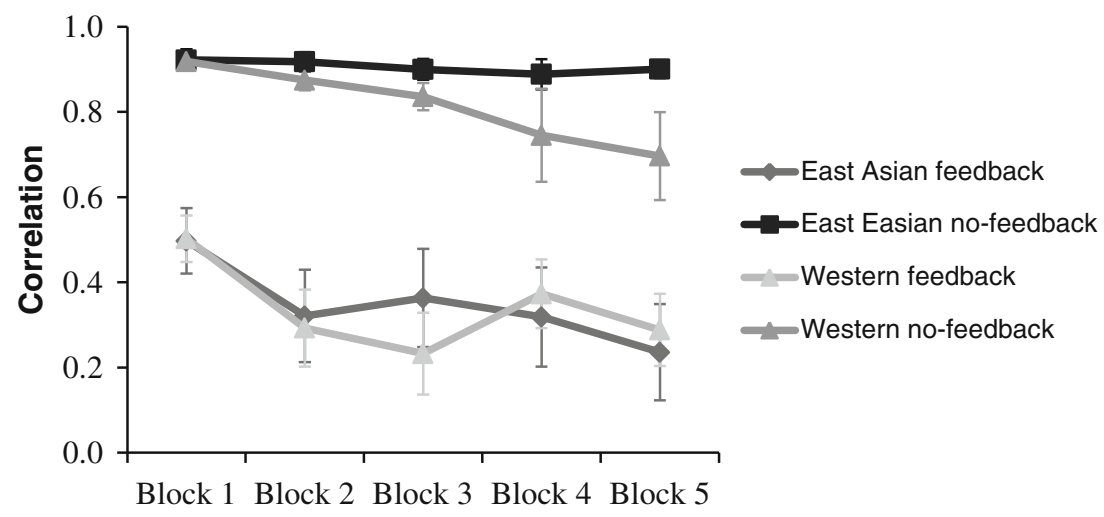




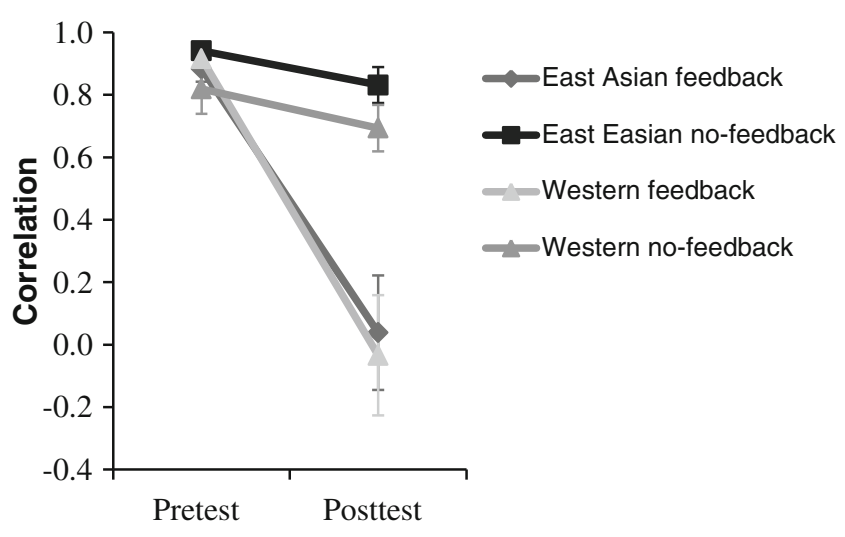

Fig. 6 Average Pearson correlations (with bars representing SEs) between signed-error scores and the size of the angle between the fins and the shaft of the Judd figures, as a function of culture and feedback for the pretest and posttest

sizeable illusory bias (i.e., larger than $1.0 \mathrm{~mm}$ ). This suggests that this subset of participants may have continued exploiting the same nonspecifying informational variable, but might have learned to scale the information differently to perception. That is, rather than attunement, the feedback in the practice blocks may have evoked calibration (see the Discussion below, in which we also consider an alternative explanation). This scaling or calibration was adjusted to the larger Judd figures presented during practice, but would then be inappropriate (i.e., too large) for the smaller Judd figures presented in the posttest. As a consequence, a negative illusory bias occurred. This may be analogous to the negative aftereffects typically observed after a recalibration of perception induced by wedge prisms (e.g., Redding \& Wallace, 1997; see also van der Kamp, Bennett, Savelsbergh, \& Davids, 1999).

\section{Discussion}

In the present study, we examined whether individual differences in perception of the midpoint of the shafts of Judd drawings point to differences in the pickup of informational variables. Two sources for these individual differences were addressed: culture and practice. Although the effect was not ubiquitous, the study revealed that East Asians tend to show a larger illusory bias in their midpoint perception than do Westerners, which is consistent with prior observations that perception is relatively context-dependent in East Asian as compared to Western persons (see Ames \& Fiske, 2010; Nisbett, 2003, 2007). Nonetheless, this enhanced illusory bias only became apparent after repetitive presentations of Judd figures. The illusory biases in East Asians and Westerners were of equal magnitude in the pretest. The two groups differed, however, in their propensity to learn to perceive the midpoint of the line. First, although both the East Asians and Westerners clearly profited from feedback in order to reduce the illusory bias (see Brosvic \& Cohen, 1988), the Westerners did so at a much faster rate. Even so, this difference between the two feedback groups had disappeared in the posttest. In
Fig. 7 Pearson correlations between signed-error scores and the size of the angle between the fins and the shaft of the Judd figures on the pretest and posttest, for a subset of the individual participants in the East Asian feedback group (top left panel), the East Asian nofeedback group (top right panel), the Western feedback group (bottom left panel), and the Western no-feedback group (bottom right panel)
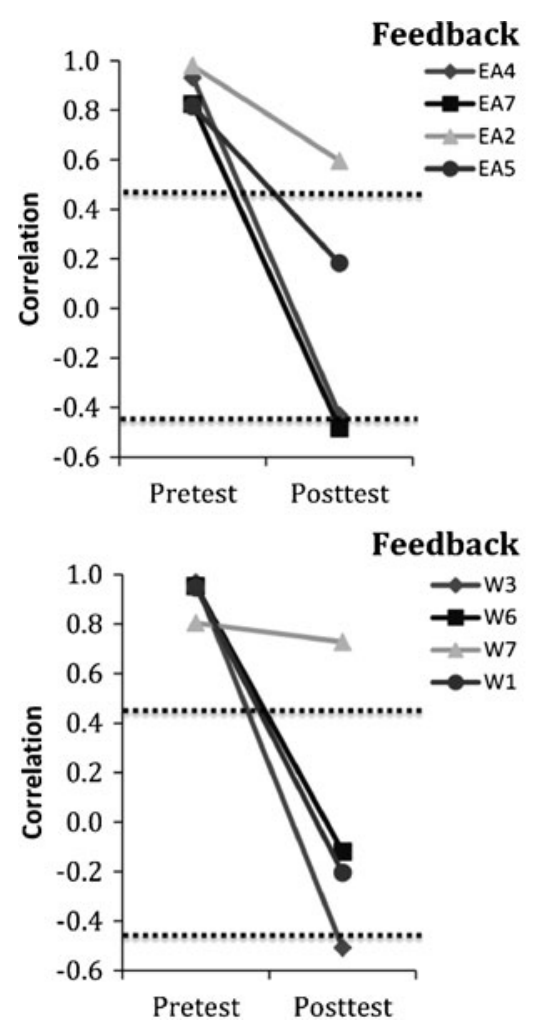
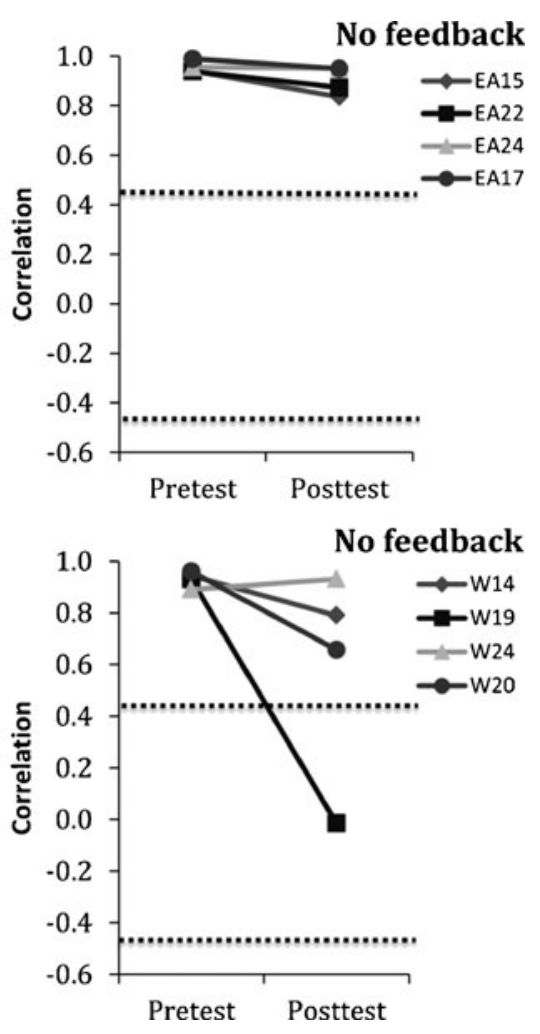
addition, even the mere practice with (or possibly exposure to) the Judd figure in the absence of feedback already resulted in a significant reduction of the illusory bias (see also Eysenck \& Slater, 1958; Girgus, Coren, Durant, \& Porac, 1975; Judd, 1902; Predebon, 1998, 2006; Schiano \& Jordan, 1990). Intriguingly, however, this was only observed among Westerners; the East Asian participants did not reduce their illusory biases during practice without feedback. Thus, East Asians were more susceptible to illusory aspects of the Judd drawing than were Westerners when they did not receive feedback about the accuracy of their perceptions. In sum, the results of the present study suggest that the differences in perception related to culture may partly be explained by different propensities for perceptual learning. Differences in learning propensity or capacity have been demonstrated earlier-for instance, when comparing young adults and elderly people (Withagen \& Caljouw, 2011). The present study demonstrates that culture (at least for the simple illusory drawings used here) can also affect the propensity for perceptual learning.

It is often presumed that the social-cultural and built environments are important determinants for cultural differences in perception (Nisbett, 2003, 2007; Nisbett \& Miyamoto, 2005; Segall et al., 1966). For example, Miyamoto, Nisbett, and Masuda (2006) counted that Japanese relative to American cityscapes were more complex and contained more objects. In a subsequent experiment, these perceptual environments were used to prime Japanese and American persons, who then participated in an allegedly unrelated change blindness experiment. Participants primed with the Japanese scenes detected more contextual changes than did participants primed with the American scenes, over and above the expected difference as a function of cultural background. This shows that cultural environments bring about different perceptions. Accordingly, Goh et al. (2007) showed that the perceptual differences between East Asians and Westerners in attention for objects versus context increased with age. In other words, many years of cultural immersion seems to lead the perceptual system to adapt to the specifics of its environment.

From an ecological perspective, this would mean for the present study that, relative to Westerners, East Asian people are more likely to be attuned to the nonspecifying informational variables that are related to illusion-inducing contextual aspects of the Judd figure (or, alternatively, are less likely to attune to specifying variables) and differ in the ease with which they converge to more useful informational variables. (In fact, this difference might turn out to be enhanced in older adults; see Goh et al., 2007; Withagen \& Caljouw, 2011.) Clearly, the individual analyses of information pickup show that a considerable number of participants changed in the informational variables that they used. In the pretest, all participants exploited nonspecifying informational variables, resulting in a systematic illusory bias in midpoint perception. By the end of practice in the final block, however, the perceptions of slightly more than half of the participants who received feedback were not correlated anymore with this nonspecifying variable; the concomitant reduction in illusory bias suggested that they had converged to a specifying informational variable. Among the participants who did not receive feedback, two spontaneously converged to more useful variables. However, the posttest results indicated that the change in variable use was relatively permanent for only half of the participants who changed during practice. The remaining participants returned to, or continued (if they had not changed variable use during practice), using the nonspecifying informational variable that induced the illusory bias. Possibly, a similar thing happened in the recent work of Jacobs et al. (2011), who found only small changes toward a stronger reliance on specifying variables. Because these authors did not report individual trajectories, this may equally well point to only few participants changing their information pickup, rather than to a small change across all or most participants. Intriguingly, it turned out that some of the participants who still used the nonspecifying informational variable by the end of the present study actually demonstrated a negative illusory bias in the posttest. We will briefly return to this finding below, when we discuss calibration.

Although changes in information pickup can account (at least to some extent) for the reduction in illusory bias, they less easily explain the observed cultural differences. That is, in the final practice block and posttest, respectively, six East Asians and nine Westerners (including two from the nofeedback group) and two East Asians and five Westerners (one from the no-feedback group) did change variable use relative to the pretest. These numbers are too small to unambiguously argue for culture differences in attunement; yet, they also do not contradict the idea that East Asians are less inclined to change in variable use. It should be pointed out, however, that the analysis conducted here to test for a change in variable use was not very fine-grained, and may have lacked power to detect changes. In this respect, we may have underestimated the degree to which attunement did occur. For instance, only one nonspecifying informational variable was scrutinized, while East Asian participants (and the Westerners as well, of course) may have changed between nonspecifying variables. Moreover, the variable did not vary to a great extent, which weakens the strength of the correlational analyses.

An alternative option is that East Asians and Westerners did use the same nonspecifying informational variable, but, due to differences in calibration, the errors in the perceived midpoints remained bigger among the East Asian observers. That is, perception not only requires the pickup of an informational variable, but also an appropriate metrical scaling or calibration of perception to that variable (Cabe \& Wagman, 
2010; Wagman, McBride, \& Trefzger, 2008; Withagen \& Michaels, 2005). Consequently, the individual differences in perception (and learning) may also imply differences in calibration among the perceivers. Perceivers may pick up the same nonspecifying informational variable, leading to qualitatively similar patterns of errors, but still yield errors of different magnitudes, dependent on calibration. In fact, the present analyses suggest that practice induced calibration among some of the participants who received feedback. Yet, although this calibration of the nonspecifying variable resulted in more accurate perception during practice, it was nonadaptive relative to the figures presented in the posttest (cf. Kennedy, Green, Nicholls, \& Liu, 1992). In the posttest, these participants showed negative correlations between the (same) nonspecifying variable and perceptual error, indicating that the relationship turned into its inverse. As a result, the illusory bias was of the same magnitude, but negative ${ }^{8}$ : A Judd drawing with the fins directed leftward resulted in an error toward the left, instead of toward the right, as it typically does (for a similar finding, see Köhler \& Fishback, 1950). Five East Asian and four Western participants in the feedback groups showed this pattern. However, the present experiment did not allow for (and was not designed for) evaluating the degree to which the cultural differences in the magnitudes of error observed during practice (especially between the nofeedback groups) also imply differences in the metric scaling of perception to the used variable. This would require the use of drawings with a greater variation in line length (see Cabe \& Wagman, 2010, for an excellent tutorial on measuring calibration).

Our account is openly indebted to ecological psychology, yet this does not, of course, preclude that the observed reduction in illusory bias could also be cast within a cognitive account (see, e.g., Gregory, 1963, 1997). Accordingly, the estimation of the midpoint of the line of a Judd figure could result from an unconscious inference process that constructs a representation of the world from retinal cues. This inference process, which obviously did not evolve to enable picture perception, is not always appropriate for geometrical drawings such as the Judd figure- hence, the illusion. However, since the inference process is supposed to be automatic, hardwired, and cognitively impenetrable, the perception itself cannot be amended with practice. Rather, the observer would develop a deliberate strategy to tweak the perceived midpoint that was indicated on the basis of the received feedback and the properties of the figure (e.g., the direction of the fins). In other words, the observer does not learn to perceive the midpoint

\footnotetext{
${ }^{8}$ This also explains the paradox that the average illusory biases for the feedback groups were close to zero in the posttest, despite many of the individual participants using nonspecifying informational variables or inappropriately scaling the perception to the exploited informational variable. That is, the positive and negative illusory biases of the individual participants canceled each other out.
}

differently, but learns to adjust the response on the basis of the perception. Notice the distinction with ecological psychology, in which changes in variable use directly affect perception. The negative illusory bias observed for some of the participants can be neatly reconciled with the use of a deliberate cognitive strategy; in the posttest, these participants continued to adopt the strategy, but were unaware that the smaller figures necessitated a readjustment of the response. Yet, it is not clear from such an account how to address the reduction in illusory bias for the no-feedback groups. The decrement without feedback is in itself not a new finding (see, e.g., Judd, 1902). It is often explained by calling for a recalibration of the cognitive process that causes the illusion, on the basis of (intrinsic) feedback from bottom-up processes, such as eye movement corrections occurring during the mere visual exposure to a geometrical drawing (Coren \& Girgus, 1978; Girgus et al., 1975; Predebon, 1998, 2006; cf. Schiano \& Jordan, 1990). Nevertheless, both variants of the cognitive account have difficulty explaining why the reduction in illusory bias would only occur among Westerners and not in East Asians.

In conclusion, we demonstrated variability among perceivers of different cultures in judging the midpoints of the lines of Judd figures, particularly with respect to the propensity of participants to improve perceptual accuracy after repeated practice and feedback. Relatively permanent improvements due to practice (at least for some participants) could be attributed to changes in variable use. However, future research will be necessary in order to more unambiguously show that the cultural differences were indeed due to variability in the pickup of informational variables and/or to calibration of the perception to the detected informational variable.

Author Note We thank Carianne van Dorst for pointing us toward cultural studies in perception, and Ludger van Dijk for his assistance in carrying out the experiment. We are also indebted to the reviewer and editor for their constructive comments.

\section{References}

Ames, D. L., \& Fiske, S. T. (2010). Cultural neuroscience. Asian Journal of Social Psychology, 13, 72-82.

Brosvic, G. M., \& Cohen, B. D. (1988). The horizontal-vertical illusion and knowledge of results. Perceptual and Motor Skills, 67, 463-469.

Cabe, P. A., \& Wagman, J. B. (2010). Characterizing perceptual learning using regression statistics: Development of a perceptual calibration index. The American Journal of Psychology, 123, 253-267.

Chemero, A. (2009). Radical embodied cognitive science. Cambridge, MA: MIT Press.

Coren, S., \& Girgus, J. S. (1978). Seeing is deceiving: The psychology of visual illusions. Hillsdale, NJ: Erlbaum.

Danks, D., \& Rose, D. (2010). Diversity in representations; uniformity in learning. The Behavioral and Brain Sciences, 33, 30-31. 
Dawson, J. L., Young, B. M., \& Choi, P. (1973). Developmental influences on geometric illusion susceptibility among Hong Kong Chinese children. Journal of Cross-Cultural Psychology, 4, 49-74.

de Fockert, J., Davidoff, J., Fagot, J., Parron, C., \& Goldstein, J. (2007). More accurate size contrast judgments in the Ebbinghaus illusion by a remote culture. Journal of Experimental Psychology: Human Perception and Performance, 33, 738-742. doi:10.1037/ 0096-1523.33.3.738

Eysenck, H. J., \& Slater, P. (1958). Effects of practice and rest on fluctuations in the Müller-Lyer illusion. British Journal of Psychology, 49, 246-256.

Gibson, J. J. (1966). The senses considered as perceptual systems. Boston, MA: Houghton Mifflin.

Gibson, J. J. (1979). The ecological approach to visual perception. Boston, MA: Houghton Mifflin.

Girgus, J. S., Coren, S., Durant, M., \& Porac, C. (1975). The assessment of components involved in illusion formation using a long-term decrement procedure. Perception \& Psychophysics, 18, 144-148.

Goh, J. O., Chee, M. W., Tan, J. C., Venkatraman, V., Hebrank, A., Leshikar, E. D., et al. (2007). Age and culture modulate object processing and object-scene binding in the ventral visual area. Cognitive, Affective, \& Behavioral Neuroscience, 7, 44-52. doi:10.3758/CABN.7.1.44

Greene, E., \& Nelson, B. (1997). Evaluating Müller-Lyer effects using single-fin configurations. Perception \& Psychophysics, 59, 292-312.

Gregory, R. L. (1963). Distortion of space as inappropriate constancy scaling. Nature, 199, 678-680.

Gregory, R. L. (1997). Knowledge in perception and illusion. Philosophical Transactions of the Royal Society B, 352, 1121-1127.

Henrich, J., Heine, S. J., \& Norenzayan, A. (2010). The weirdest people in the world. The Behavioral and Brain Sciences, 33, 1-23.

Ingold, T. (2000). The perception of the environment: Essays in livelihood, dwelling and skill. London, UK: Routledge.

Jacobs, D. M., Ibáñez-Gijón, J., Díaz, A., \& Travieso, D. (2011). On potential-based and direct movements in information spaces. Ecological Psychology, 23, 123-145.

Jacobs, D. M., \& Michaels, C. F. (2007). Direct learning. Ecological Psychology, 19, 321-349.

Jacobs, D. M., Michaels, C. F., \& Runeson, S. (2000). Learning to perceive the relative mass of colliding balls: The effects of ratioscaling and feedback. Perception \& Psychophysics, 62, 1332-1340.

Jacobs, D. M., Runeson, S., \& Michaels, C. F. (2001). Learning to visually perceive the relative mass of colliding balls in globally and locally constrained task ecologies. Journal of Experimental Psychology: Human Perception and Performance, 27, 10191038. doi:10.1037/0096-1523.27.5.1019

Ji, L. Y., Peng, K., \& Nisbett, R. E. (2000). Culture, control, and perception of relationships in the environment. Journal of Personality and Social Psychology, 78, 943-955.

Judd, C. H. (1902). Practice and its effects on the perception of illusion. Psychological Review, 9, 27-39.

Kennedy, J. M., Green, C. D., Nicholls, A., \& Liu, C. H. (1992). Illusions and knowing what is real. Ecological Psychology, 4, $153-172$.

Köhler, W., \& Fishback, J. (1950). The destruction of the Müller-Lyer illusion in repeated trials: II. Satiation patterns and memory traces. Journal of Experimental Psychology, 4, 389-410.

Massaro, D., \& Anderson, N. (1970). A test of a perspective theory of geometrical illusions. The American Journal of Psychology, 83, $565-575$.

Menger, R., \& Withagen, R. (2009). How mechanical context and feedback jointly determine the use of mechanical variables in length perception by dynamic touch. Attention, Perception, \& Psychophysics, 71, 1862-1875. doi:10.3758/APP.71.8.1862
Michaels, C. F., \& de Vries, M. M. (1998). Higher order and lower order variables in the visual perception of relative pulling force. Journal of Experimental Psychology: Human Perception and Performance, 24, 526-546. doi:10.1037/0096-1523.24.2.526

Miyamoto, Y., Nisbett, R. E., \& Masuda, T. (2006). Culture and the physical environment. Psychological Science, 17, 113119.

Nisbett, R. E. (2003). The geography of thought. New York, NY: Free Press.

Nisbett, R. E. (2007). Eastern and western ways of perceiving the world. In Y. Shoda, D. Cervone, \& G. Downey (Eds.), Persons in context: Building a science of the individual (pp. 62-83). New York, NY: Guilford Press.

Nisbett, R. E., \& Miyamoto, Y. (2005). The influence of culture: Holistic versus analytic perception. Trends in Cognitive Sciences, 9, 467-473. doi:10.1016/j.tics.2005.08.004

Predebon, J. (1998). Decrement of the Brentano Müller-Lyer illusion as a function of inspection time. Perception, 27, 183-192.

Predebon, J. (2006). Decrement of the Müller-Lyer and Poggendorf illusions: The effect of inspection and practice. Psychological Research, 70, 384-394.

Pressey, A. W. (1974). Effect of size of angle on the ambiguous MüllerLyer illusion. Acta Psychologica, 38, 401-404.

Redding, G. M., \& Wallace, B. (1997). Adaptive spatial alignment. Hillsdale, NJ: Erlbaum.

Reed, E. S. (1996). Encountering the world: Toward an ecological psychology. New York, NY: Oxford University Press.

Rivers, W. H. R. (1905). Observations on the senses of the Todas. British Journal of Psychology, 1, 321-396.

Rosengren, K. S., Savelsbergh, G. J. P., \& van der Kamp, J. (2003). Development and learning: A TASC-based perspective of the acquisition of perceptual-motor behaviors. Infant Behavior \& Development, 26, 473-494.

Runeson, S. (1988). The distorted room illusion, equivalent configurations, and the specificity of static optic arrays. Journal of Experimental Psychology: Human Perception and Performance, 14, 295-304

Runeson, S., \& Andersson, I. E. (2007). Achievement of specificational information usage with true and false feedback in learning a visual relative-mass discrimination task. Journal of Experimental Psychology: Human Perception and Performance, 33, 163-182. doi:10.1037/0096-1523.33.1.16

Schiano, D. J., \& Jordan, K. (1990). Müller-Lyer decrement: Practice or prolonged inspection? Perception, 19, 307-316.

Segall, H. H., Campbell, D. T., \& Herskovits, M. J. (1966). The influence of culture on visual perception. Indianapolis, IN: Bobbs-Merrill.

van der Kamp, J., Bennett, S. J., Savelsbergh, G. J. P., \& Davids, K. (1999). Timing a one-handed catch: II. Adaptation to telestereoscopic viewing. Experimental Brain Research, 129, 369-377.

van der Kamp, J., de Wit, M., \& Masters, R. S. W. (2012). Left, right, left, right, eyes to the front! Müller-Lyer bias in grasping is not a function of hand used, hand preferred or visual hemifield, but foveation does matter. Experimental Brain Research, 218, 91-98.

Wagman, J. B., McBride, D. M., \& Trefzger, A. J. (2008). Perceptual experience and posttest improvements in perceptual accuracy and consistency. Perception \& Psychophysics, 70, 1060-1067. doi:10.3758/PP.70.6.1060

Warren, W. H. (2005). Direction perception: The view from here. Philosophical Topics, 33, 335-361.

Withagen, R. (2004). The pickup of nonspecifying variables does not entail direct perception. Ecological Psychology, 16, 237253.

Withagen, R., \& Caljouw, S. R. (2011). Aging affects attunement in perceiving length by dynamic touch. Attention, Perception, \& Psychophysics, 7, 1216-1226. doi:10.3758/APP.71.1.64 
Withagen, R., \& Chemero, A. (2009). Naturalizing perception: Developing the Gibsonian approach along evolutionary lines. Theory and Psychology, 19, 363-389.

Withagen, R., \& Michaels, C. F. (2005). The role of feedback information for calibration and attunement in perceiving length by dynamic touch. Journal of Experimental Psychology: Human Perception and Performance, 31, 1379-1390. doi:10.1037/ 0096-1523.31.6.1379
Withagen, R., \& van der Kamp, J. (2010). Towards a new ecological conception of perceptual information: Lessons from a developmental systems perspective. Human Movement Science, 29, 149163.

Withagen, R., \& van Wermeskerken, M. (2009). Individual differences in learning to perceive length by dynamic touch: Evidence for variation in perceptual learning capacities. Attention, Perception, \& Psychophysics, 71, 64-75. doi:10.3758/APP.71.1.64 